\title{
Calidad de vida relacionada con la salud de los pacientes con insuficiencia cardiaca crónica sistólica en España: resultados del estudio VIDA-IC
}

Comín J*, Anguita M, Formiga F, Almenar L, Crespo-Leiro MG, Manzano L, Muñiz J, Chaves J, de Frutos T, Enjuanes C, en representación de los investigadores del estudio multicéntrico VIDA-IC (Calidad de VIDA e Insuficiencia Cardiaca en España: situación actual).

\section{RESUMEN}

Introducción y objetivos: La calidad de vida relacionada con la salud (CVRS) está afectada en pacientes con insuficiencia cardiaca (IC). Existe poca información sobre los factores clínicos asociados a mala CVRS en la población española con IC y con esta finalidad se diseñó este estudio. Métodos: Evaluación multicéntrica transversal de la CVRS mediante cuestionarios específico (Kansas City CardiomyopathyQuestionnaire) y genérico (EuroQoL 5 dimensiones) en 1037 pacientes ambulatorios consecutivos con IC sistólica. Resultados: Los pacientes con peor CVRS presentaron en su mayoría datos asociados a peor pronóstico y mayor severidad de la enfermedad. En base a una comparación externa, los pacientes con IC presentaron una mayor incidencia de limitaciones en movilidad, dolor/disconfort y ansiedad/depresión en comparación con la población general y con pacientes con otraspatologías crónicas. La correlación entre la puntuación global de ambos cuestionarios fue muy alta ( $r$ de Pearson=0,815, $p<0,001)$. En la regresión lineal multivariada se observó que una mayor edad ( $\beta$ estandarizada $=-0,2 ; p=0,03$ ), sexo femenino ( $\beta$ estandarizada $=-10,3 ; p<0,001$ ), peor clase funcional ( $\beta$ estandarizada=-20,4; $p<0,001$ ), mayor comorbilidad según índice de Charlson ( $\beta$ estandarizada $=-1,2 ; p=0,005)$ y el ingreso reciente por insuficiencia cardiaca $(\beta$ estandarizada $=6,28 ; p=0,006$ ) fueron factores predictores independientes de peor CVRS.

\section{Conclusión:}

Los pacientes con IC presentan una gran afectación de su CVRS en relación a la población general española y a otras patologías crónicas. Factores como el sexo femenino, la edad avanzada, la comorbilidad, los síntomas avanzados y la hospitalización reciente son factores determinantes en la CVRS de estos pacientes.

Palabras Clave:Insuficiencia cardíaca, calidad de vida relacionada con la salud, cuestionarios de calidad de vida específicos y genéricos, vida real o práctica clínica habitual 


\section{ABSTRACT}

Background and Objectives: Health-related quality of life (HRQOL) is impaired in patients with heart failure (HF). There is little information on the clinical factors associated with poor HRQOL in the Spanish population with systolic HF and this study was designed for this purpose. Methods: A descriptive, crossectional, multicenter evaluation of HRQOL using specific (Kansas City Cardiomyopathy Questionnaire) and generic questionnaires (EuroQoL 5 dimensions) in 1037 consecutive outpatients with systolic HF. Results: Patients with poorer HRQOL had clinical characteristics associated with poor prognosis and more severe disease. Based on an external comparison, the patients with HF had a higher incidence of limitations in mobility, pain / discomfort and anxiety / depression compared with the general population and patients with other chronic diseases. The correlation between the overall score of both questionnaires was very high $(r$ Pearson $=0.802, p<0.001)$. In multivariate linear regression analysis, older age (standardized $\beta=-0.2 ; p=0.03$ ), female sex (standardized $\beta=-10.3 ; p<0.001$ ), worse functional class (standardized $\beta=-20.4 ; p<0.001$ ), higher Charlson comorbidity index (standardized $\beta=-1.2, p=0.005$ ) and recent hospitalization for heart failure (standardized $\beta=$ $6.28 ; p=0.006$ ) were independent predictors of poorer $\mathrm{HRQOL}$ in these patients.

Conclusion:

Patients with HF suffer a great impairment in HRQOL compared to the Spanish general population and to patients with other chronic conditions. Factors such as female sex, older age, comorbidity, advanced symptoms and recent hospitalization are determinants of HRQOL in these patients.

Key words: Heart Failure, health related quality of life, generic and specific questionnaires of quality of life, real world or usual care 
Lista de Abreviaturas (6)

AbbreviationList (6)

\begin{tabular}{|c|c|c|c|}
\hline \multicolumn{2}{|c|}{ Español } & \multicolumn{2}{|l|}{ English } \\
\hline ICC & $\begin{array}{l}\text { Insuficiencia } \\
\text { Cardiaca Crónica }\end{array}$ & $\mathrm{CHF}$ & Chronic Heart Failure \\
\hline CVRS & $\begin{array}{l}\text { Calidad de vida } \\
\text { relacionada con la } \\
\text { salud }\end{array}$ & HRQoL & Health-related quality of life \\
\hline $\mathrm{KCCQ}$ & $\begin{array}{l}\text { Cuestionario de } \\
\text { calidad de vida } \\
\text { específico en } \\
\text { cardiomiopatías } \\
\text { Kansas City }\end{array}$ & $\mathrm{KCCQ}$ & $\begin{array}{l}\text { Kansas City } \\
\text { CardiomyopathyQuestionnaire }\end{array}$ \\
\hline EQ 5D & $\begin{array}{l}\text { Cuestionario } \\
\text { general de calidad } \\
\text { de vida Euro QoL } \\
\text { 5D }\end{array}$ & EQ 5D & Euro Qol 5D Questionnaire \\
\hline EAV & $\begin{array}{l}\text { Escala Analógica } \\
\text { Visual }\end{array}$ & VAS & Visual Analogicscale \\
\hline NYHA & $\begin{array}{l}\text { Clase functional } \\
\text { según New York } \\
\text { Heart Association }\end{array}$ & NYHA & $\begin{array}{l}\text { Class New York Heart } \\
\text { Association }\end{array}$ \\
\hline
\end{tabular}




\section{INTRODUCCIÓN}

Los pacientes con insuficiencia cardíaca crónica (ICC) sufren de un marcado deterioro de la calidad de vida relacionada con la salud (CVRS) en comparación con la población normal y con pacientes afectadospor otras patologías crónicas ${ }^{1}$. Por ello, la mejora de la CVRS se considera como uno de los principales objetivos de la gestión integral de los pacientes con ICC ${ }^{2-4}$

En estos pacientes, la CVRS es una medida multidimensional con buena correlación con la severidad de la enfermedad ${ }^{5}$, aporta información pronóstica independiente y permite evaluaciones de coste-eficacia a la hora de implementar nuevas opciones terapéuticas ${ }^{6,7}$.

El deterioro de la CVRS en pacientes con ICC se refleja en las dimensiones que capturan información sobre la limitación funcional con una especial repercusión sobre los dominios que informan sobre la movilidad o actividades cotidianas ${ }^{8}$.

Diversos autores han abordado en qué medida los pacientes con ICC ven su CVRS afectada en relación a la población general o respecto a pacientes con otras patologías crónicas, qué dimensiones o dominios de la CVRS se hallan más afectados y cuáles son los factores clínicodemográficos que influyen. Sin embargo, la información en este ámbito es escasa en lo que respecta a la población con IC en España dado que lo publicado hasta la fecha en este terreno proviene de sub-estudios de ensayos clínicos o de estudios realizados en otros ámbitos geográficos y socio-culturales distintos al nuestro por lo que se desconoce si son completamente trasladables a nuestra realidad ${ }^{9,10}$.

Así, los objetivos de este análisis pre-especificado del Estudio VIDA-ICcuyos primeros resultados se publicaron en $2014^{11}$ fueron, 1) determinar los factores clínico-demográficos asociados a la CVRS en pacientes con ICCy disfunción sistólica del ventrículo izquierdo seguidos en consultas de Cardiología o Medicina Interna, 2) evaluar las dimensiones más afectadas en estos y 3 ) explorar la existencia de un gradiente de puntuaciones globales y por 
dominios específicos en los instrumentos de CVRS entre los pacientes de nuestro estudio y la población general española o los pacientes con otras patologías crónicas evaluados en nuestro país.

\section{MÉTODOS}

\section{Diseño del Estudio}

El estudio VIDA-IC es un estudio nacional, observacional descriptivo transversal realizado por 115 especialistas (63.2\% cardiólogos y 36.8\% medicina interna) de octubre 2011 a enero 2012.cardiólogos y medicina interna) de toda España que incluyeron pacientes consecutivos que acudían a su consulta con $I C C^{11}$.Los objetivos del estudio fueron evaluar el nivel de concordancia entre medidas específicas y genéricas de CVRS en estos pacientes, estudiar los factores determinantes del nivel de CVRS y contextualizar los datos obtenidos con medidas genéricas de CVRS entre nuestros pacientes y la población general o pacientes con otras cronicidades en España. Este último objetivo se efectuó en base a una comparación externa con los datos de calidad de vida genérica disponibles para población general española y población española con enfermedades crónicas a partir de la literatura y de las fuentes públicas de las encuestas nacionales de salud.

El protocolo del estudio fue aprobado por Comité de Ética e Investigación Clínica del Instituto Hospital del Mar de Investigaciones Médicas (IMIM) de Barcelona. Todos los pacientes dieron su consentimiento informado por escrito antes de la inclusión en el estudio.

\section{Población del Estudio y Criterios de Inclusión y Exclusión}

Se incluyeron a pacientes consecutivos que acudían a la consulta ambulatoria especializada (Cardiología o Medicina Interna) que cumplieran los siguientes criterios de inclusión: edad $\geq 18$ años, diagnóstico de ICC con disfunción sistólica (fracción de eyección ventricular izquierda [FEVI] $\leq 40 \%$ ) en los últimos 12 meses y situación clínica estable. Fueron criterios de exclusión: estar a la espera de trasplante cardíaco o corrección de lesiones valvulares, incapacidad de valorar y rellenar los cuestionarios de CVRS, enfermedad extra-cardíaca con expectativa de vida inferior a 1 año, ingreso hospitalario de origen no cardiovascular en el mes previo a la inclusión, hospitalización en el momento de la inclusión. La inclusión de los pacientes se estratificó en función de la presencia o ausencia de ingreso previo reciente por IC $(<1$ mes y $>6$ 
meses sin ingreso reciente por IC) en un razón 1:1 para cada uno de los investigadores reclutadores. La información correspondiente a los datos basales se obtuvo en los pacientes elegibles tras el consentimiento informado si estaban estables sin signos de descompensación aguda a partir de los pacientes o las historias clínicas.

\section{Evaluación de los resultados en salud centrados en el paciente: Calidad de Vida}

A todos los pacientes del estudio se les solicitó que cumplimentaran de forma autoadministrada el Kansas City CardiomyopathyQuestionnaire (KCCQ) ${ }^{12}$ y el EuroQol 5D (EQ$5 D)^{13}$. EI KCCQ es un instrumento específico para IC, compuesto por 23 ítems que componen 7dimensiones. La puntuación de cada dimensión tiene una gama teórica de 0 a 100, siendo 100 el mejor estado. Además, se calculan 3 puntuaciones resumen: el sumario de síntomas (SS) como resultado de la suma de la frecuencia y gravedad de los síntomas (excluyendo estabilidad), sumario clínico (SC) como resultado de la suma de la puntuación de los dominios limitación física y de síntomas y el sumario general (SG) como del SC y de los dominios de calidad de vida y limitación social. El EuroQol 5D (EQ-5D, es un instrumento genérico. Consta de una escala analógica visual (EAV, auto-evaluación de la salud general) y 5 dominios (movilidad, autocuidado, actividades cotidianas, dolor/malestar y ansiedad/depresión). Para la EAV, el rango es de 0 (peor estado) a 100 (mejor estado). Para el resto de dimensiones los resultados se pueden expresar como índice resumen general (índice EQ-5D) o pueden ser expresadas en \% de pacientes que reportan algún tipo de problema en cada una de las dimensiones. Ambas escalas han sido validadas en España ${ }^{8,13}$.

Para la comparación del impacto en la CVRS de los pacientes con ICC incluidos en el estudio respecto a personas de la población general española y otras personas afectadas por otras patologías crónicas se tomaron los datos sumario resumen de la EAV y de las 5 dimensiones del EQ-5D procedentes de datos publicados de la última Encuesta Nacional de Salud en población general ${ }^{14} \mathrm{y}$ de publicaciones que evaluaron la CVRS mediante EQ-5D en pacientes españoles afectadospor diversas patologías crónicas ${ }^{15-18}$.

\section{Análisis Estadístico}

Las variables continuas se expresan como media \pm desviación estándar y las variables categóricas como $\mathrm{n}$ (porcentaje). Las comparaciones entre los grupos con CVRS preservada y 
afectada se realizaron mediante las pruebas de $\mathrm{chi}^{2}$ de la t de Student (o la prueba U de MannWhitney según fuera aplicable) en el caso de variables categóricas y cuantitativas respectivamente..El nivel de correlación entre las puntuaciones globales del KCCQ y el EQ-5D se evaluó mediante los coeficientes de correlación Rho de Spearman y R de Pearson. Para la evaluación de los factores clínicos y demográficos asociados a la CVRS se llevaron a cabo modelos univariantes de regresión logística y modelos de regresión lineal univariante, donde las variables dependientes consideradas fueron el SG del KCCQ, índice del EQ-5D y la EAV, y las variables independientes fueron ciertos factores demográficos y clínicos estudiados en este trabajo.. A partir de estos últimos se realizaron diversos modelos exploratorios de regresión lineal multivariante con el método de pasos atrás para determinar qué factores mantenían una asociación independiente con los resultados en salud centrados en el paciente. Un valor de $p<$ 0,05 fue considerado estadísticamente significativo. Los análisis se realizaron con SPSSv18 y Stata v11.

\section{RESULTADOS}

Para este estudio se incluyeron 1037 pacientes con insuficiencia cardiaca y disfunción sistólica del ventrículo izquierdo. El 63,2\% de los pacientes fueron incluidos por un cardiólogo y el $36,8 \%$ por un especialista en medicina interna. Para este análisis se dispuso del siguiente número de cuestionarios de CVRS completos: 1037 para KCCQ, 1020 para EAV y 1009 para EQ-5D. Las características de los pacientes incluidos se presentan en la tabla 1. La mediana de edad fue de 72 años con un rango intercuartílico entre 64 (percentil 25) y 78 años (percentil 75)y hubo predominio de varones. Aproximadamente, la mitad de pacientes tenían IC de origen isquémico y se hallaban en una clase funcional NYHA III-IV. En general, los pacientes con peor CVRS en base al KCCQ presentaron datos asociados a peor pronóstico y mayor severidad de ICC.

En comparación a la población general de referencia (figura 1), los pacientes del estudio, refirieron mayor tasa de limitaciones en todas las dimensiones del EQ-5D. En algunas dimensiones tales como movilidad, dolor/disconfort y ansiedad depresión, los pacientes con IC refirieron mayor incidencia de limitaciones que los pacientes con patologías crónicas tales como la diabetes, cáncer o Alzheimer. Es importante destacar que los pacientes con IC y clase funcional III-IV, que representaron prácticamente la mitad de los pacientes de nuestro estudio, 
refirieron limitaciones similares o superiores en la mayoría de dimensiones exploradas que los pacientes con antecedentes de ictus o pacientes con insuficiencia renal crónica en diálisis. En el análisis de las puntuaciones medias de la EAV se hallaron similares resultados. El conjunto de pacientes con ICC del estudio manifestaron un estado de salud general percibido medido con la EAV peor que la población general, los pacientes con enfermedad pulmonar obstructiva crónica o cáncer y similar al reportado por los pacientes diabéticos o con hipertensión pulmonar. En este sentido, en pacientes con ICC en clase funcional NYHA III-IV la puntuación media de la EAV fue menor y por lo tanto se tradujo en un peor estado general de salud percibido incluso comparado con los pacientes con antecedentes de ictus, Alzheimer o en diálisis.

En la tabla 2 se presentan las puntuaciones medias de cada una de los subdominios del KCCQ, de sus puntuaciones sumarias, las puntuaciones medias del índice EQ-5D y de la EAV, así como el porcentaje de pacientes que refirieron algún grado de limitación en cada una de las dimensiones del EQ-5D. Como era de esperar, los pacientes con peor nivel de CVRS puntuaron peor en todos estos ítems. Es interesante que ítems como la autoeficacia o la estabilidad de síntomas que no computan para el sumario global, fueran significativamente peores en los pacientes con peor CVRS. De forma consistente, los pacientes con un sumario global del $\mathrm{KCCQ}<50$ puntos refirieron mayor prevalencia de problemas en las 5 dimensiones del EQ-5D y presentaron medias más bajas para el índice de este cuestionario y en la EAV.

Para estudiar la relación que puede existir entre diferentes variables de los cuestionarios de CVRS utilizados en el presente estudio se utilizó una matriz de correlaciones (Tabla 3). La matriz de valores $\mathrm{R}$ muestra una lista multivariable horizontalmente y la misma lista verticalmente con el correspondiente coeficiente de correlación (R) (y sus intervalos de confianza correspondientes) entre cada pareja en cada celda, expresada con un número que va desde 0 a 1 (Panel A) y se acompaña de la matriz de los valores $P$ (Panel B).En la tabla 3 se muestra el análisis de la relación entre las puntuaciones de entre dimensiones del KCCQ y las puntuaciones sumarias de este cuestionario y del EQ-5D (índice y EAV). La correlación entre las puntuaciones globales del EQ-5D y el KCCQ fueron muy altas, con un coeficiente de correlación de Pearson $r=0,815$ y un coeficiente de correlación de Spearman $\rho=0,811$ y un valor de $p<0,001$ para ambos coeficientes. La matriz de correlaciones entre las dimensiones del 
KCCQ y éstas con las puntuaciones resumen del KCCQ y del EQ-5D mostraron entre sí una asociación significativa con un elevado nivel de correlación, superior a 0,6 en todos los casos en los que se puede esperar una correlación convergente. La dimensión limitación física y el sumario total de síntomas mostraron niveles de convergencia muy elevados con coeficientes superiores a 0,8 con respecto las puntuaciones globales del KCCQ. Estas correlaciones fueron de una relativa menor magnitud con respecto a la EAV y el índice EQ-5D. Las correlaciones que, aunque significativas, se mostraron más divergentes respecto al resto de dimensiones y las puntuaciones sumarias del KCCQ o del EQ-5D fueron las que miden la estabilidad de síntomas y la dimensión del auto-eficacia del KCCQ con rangos entre 0,1 y 0,2 en la mayoría de casos.

En la figura 2 y en la tabla 4 se muestran los factores clínicos asociados a un peor nivel de CVRS. En los análisis de regresión lineal múltiple (tabla 4) se observó que una mayor edad, sexo femenino, peor clase funcional, mayor comorbilidad, fueron factores predictores independientes de peor calidad de vida. El control del paciente en servicios de Cardiología se asoció de forma independiente a una mejor calidad de vida, probablemente relacionado con un mejor perfil clínico del paciente. En este sentido, estas características clínicas delimitaron diferencias significativas en las puntuaciones sumarias crudas de los instrumentos (tabla 5) y en el porcentaje de problemas reportados en cada dimensión del EQ-5D (figura 3).

\section{DISCUSION}

En este estudio multicéntrico español hemos observado que los pacientes con IC con disfunción sistólicasufren afectación muy importante de su CVRS. Específicamente, en pacientes con IC y clase funcional avanzada, el nivel de CVRS es similar o incluso peor que el observado en pacientes con EPOC, hipertensión pulmonar, Alzheimer, antecedente de ictus o en diálisis ${ }^{14-20}$. La puntuación media del KCCQ sumario global en nuestro estudio y muy especialmente en el subgrupo de pacientes en NYHA III-IV estuvo por debajo de la reportada en pacientes incluidos en ensayos clínicos internacionales en el campo de la ICC ${ }^{9,21,22}$. Esto pone de relieve no solo el gran nivel de afectación de la CVRS de los pacientes con IC del mundo real sino también la distancia que existe entre las poblaciones incluidas en ensayos y las que vemos en la práctica clínica diaria ${ }^{23}$. 
Este estudio demuestra que las medidas de CVRS específicas y las genéricas tienen elevado grado de correlación. Se observaron correlaciones de mayor magnitud entre las puntuaciones globales de calidad de vida medidas con el KCCQ y las dimensiones o ítems que miden la afectación física por la enfermedad. Ello está a favor de que, fundamentalmente, son las limitaciones físicas y los síntomas relacionados con la IC (componente físico) lo que determina la merma en CVRS de estos pacientes ${ }^{8,24-26}$. Es interesante observar que la magnitud de asociación entre los ítems relacionados con la limitación física o los síntomas en el KCCQ y las medidas globales genéricas del EQ-5D mostraron correlaciones altas pero ligeramente menores a las encontradas con relación a las puntuaciones globales del KCCQ para la IC. Ello podría indicar que la CVRS de los pacientes con IC está también afectada por otros factores más allá de la limitación física impuesta por la enfermedad y que otros aspectos que no se recogen de modo tan adecuado en los instrumentos específicos para la IC, como son la afectación en la capacidad de mantener los cuidados personales, el dolor, la ansiedad o los síntomas afectivos, son igualmente relevantes en la percepción del estado de salud del paciente con $\mathrm{IC}^{6,8,9,24}$. Esto pone de relieve la importancia de evaluar la CVRS en estos pacientes usando instrumentos específicos y genéricos y subraya el carácter multidimensional de la CVRS ${ }^{8}$.

Un aspecto destacado de nuestro estudio es la evaluación de los factores clínicos determinantes de la CVRS en estos pacientes. En este sentido, es preciso destacar la originalidad de nuestro estudio dada la escasez hasta la fecha de estudios multicéntricos españoles que como el nuestro hayan evaluado en un número tan grande de pacientes con IC sistólica los factores determinantes de su CVRS. Al respecto, aquellos factores asociados a una peor evolución de la enfermedad, tales como la mayor edad, la presencia de comorbilidad, ingresos recientes o peor clase funcional se asociaron de forma independiente a una peor CVRS. Muchos de estos factores se asocian no solo a peor CVRS $S^{5,6,24-26}$ sino también a mayor riesgo de muerte $u$ hospitalización ${ }^{23}$. En este sentido, estudios previos han demostrado que la CVRS es un factor predictor independiente de estos acontecimientos clínicos ${ }^{27,28}$.

La relación entre sexo y CVRS observada en nuestro estudio podría estar relacionada con la pérdida de rol social en las mujeres debido a las limitaciones impuestas por la IC o que los instrumentos que se han diseñado para medir la CVRS de alguna manera capturen mejor esta 
información en el sexo femenino. Aunquelos análisis se ajustaron por variables de severidad, las puntuaciones de los pacientes a cargo de cardiología refirieron mejor CVRS, probablemente en relación a un mejor perfil clínico que los pacientes atendidos por medicina interna. Es probable que también estas diferencias sean debidas a factores no recogidos de forma prospectiva que definen mejor al paciente en aspectos sociales o de fragilidad.

Diversas variables comúnmente usadas para estratificar el riesgo de los pacientes tales como la FEVI, la función renal o la hemoglobina, no mostraron asociación independiente con la CVRS. Ello pone de relieve la importancia de incorporar la CVRS como medida adicional en toda evaluación de un paciente con IC dado que otras variables clínicas usadas para estratificar el riesgo no sustituyen la información que aportan los instrumentos que miden la percepción del estado de salud desde la perspectiva del paciente y nos informan de las limitaciones que sufre diferentes a las obtenidas con mediciones fisiológicas 0 biológicas $^{8}$.Como último aspecto cabe resaltar la importancia de evaluar el impacto de la IC en la CVRS entornos geográficos específicos. Concretamente, la Comisión Europea pone de relieve las diferencias existentes entre paises europeos en términos de estado salud percibido y la importancia de estudio específico de estos aspectos en cada ámbito geográfico. El presente trabajo aporta una información muy valiosa al respecto ya que ofrece nueva información sobre la CVRS en pacientes españoles con IC y permite añadir nuevos datos sobre determinantes de la CVRS que complemente lo ya publicado en pacientes de otros entornos culturales o geográficos ${ }^{29}$.

\section{LIMITACIONES}

Este estudio tiene las limitaciones propias de toda evaluación transversal, ya que no aporta información sobre los cambios longitudinales de la variable a estudio y su interrelación con los determinantes clínicos explorados. La población incluida representa un subgrupo de los pacientes que conIC y disfunción sistólica que son evaluados de forma rutinaria en el ámbito de las consultas externas de nuestros hospitales. Así pues, no es posible determinar si los resultados obtenidos son extrapolables a otro tipo de poblaciones de pacientes con IC tales como los que tienen una fracción de eyección preservada como los que no siguen controles en las consultas externas hospitalarias. En este estudio se evaluaron variables fundamentalmente 
clínicas por lo que nos disponemos de información específica sobre la influencia de variables psicosociales o de hábitos higiénico-dietéticos en términos de CVRS.

\section{CONCLUSIONES}

En este estudio multicéntrico español hemos observado que los pacientes con ICC tienen una gran afectación de su CVRS en relación a la población general y a otras poblaciones de pacientes crónicos. Se observó un alto grado de correlación entre las medidas genéricas y específicas de la CVRS. Diversos factores clínicos como la edad avanzada, el sexo femenino, la clase funcional avanzada, la presencia de un ingreso reciente y la presencia de mayor comorbilidad se asociaron a la CVRS de forma independiente a otros factores con valor pronóstico. El control del paciente en servicios de Cardiología se asoció de forma independiente a una mejor calidad de vida, probablemente relacionado con un mejor perfil clínico de los pacientes que atienden.

\section{CONFLICTO DE INTERESES}

J. Comín-Colet, M. Anguita, F. Formiga, L. Almenar, M. Crespo-Leiro, L. Manzano recibieron honorarios como miembros del comité asesor del estudio VIDA-IC. J. Muñiz ha recibido honorarios por su colaboración en el análisis estadístico independiente del estudio. J. Chaves y T. de Frutos son empleados del Departamento Médico de Pfizer S.L.U. y han colaborado en la realización del estudio VIDA-IC.

\section{AGRADECIMIENTOS}

Agradecemos a todos los investigadores y pacientes que han colaborado en la realización del estudio VIDA-IC.

El estudio cuenta con el reconocimiento y apoyo de la Sección de Insuficiencia Cardíaca y Trasplante de la Sociedad Española de Cardiología y de la Sección de Insuficiencia Cardíaca de la Sociedad Española de Medicina Interna.

El trabajo de campo lo realizó SANED y ODDS, S.L. colaboró en el análisis estadístico. 


\section{PIES DE FIGURAS}

Figura 1. Comparación del impacto en la CVRS de los pacientes con IC incluidos en el estudio respecto a la población general española ${ }^{14}$ y personas afectadas por otras patologías crónicas en España ${ }^{15-20}$.Panel $A$, porcentaje de personas y pacientes que refirieron algún tipo de limitación en cada dimensión del EQ-5D. Panel B, análisis comparativo de las puntuaciones (medias \pm desviación estándar) en la EAV del EQ-5D.

Figura 2. Factores demográficos y clínicos asociados con el nivel de CVRS percibida por los pacientes evaluada con el KCCQ (Panel A) y el EQ-5D (índice EQ-5D, panel B) y EAV del EQ5D (panel C). CVRS anormal: KCCQ sumario global <50; índice EQ-5D <0,5; EAV <50. Los análisis se realizaron mediante modelos univariantes de regresión logística binaria. QoL: calidad de vida.

Figura 3. Análisis no ajustado de las 5 dimensiones del EQ-5D en los subgrupos de pacientes que mostraron asociación independiente con la CVRS en los análisis multivariantes. Se representa el porcentaje de pacientes que presentaron algún tipo de limitación en cada una de las cinco dimensiones del EQ-5D en función del sexo (panel A), clase funcional de la NYHA (panel B), edad (panel C), índice de comorbilidad de Charlson (panel D), hospitalización reciente (panel E) y servicio clínico (Panel F). 


\section{BIBLIOGRAFIA}

1. Juenger J, Schellberg D, Kraemer S, Haunstetter A, Zugck C, Herzog W et al. Health related quality of life in patients with congestive heart failure: comparison with other chronic diseases and relation to functional variables. Heart 2002;87:235-41.

2. Anker SD, Agewall S, Borggrefe M, Calvert M, Jaime CJ, Cowie MRet al. The importance of patient-reported outcomes: a call for their comprehensive integration in cardiovascular clinical trials. Eur Heart J 2014;35:2001-9.

3. Formiga F, Chivite D, Ortega C, Casas S, Ramon JM, Pujol R. End-of-life preferences in elderly patients admitted for heart failure. QJM 2004;97:803-8.

4. Rumsfeld JS, Alexander KP, Goff DC, Jr., Graham MM, Ho PM, Masoudi FA et al. Cardiovascular health: the importance of measuring patient-reported health status: a scientific statement from the American Heart Association. Circulation 2013;127:2233-49.

5. Myers J, Zaheer N, Quaglietti S, Madhavan R, Froelicher V, Heidenreich P. Association of functional and health status measures in heart failure. J Card Fail 2006;12:439-45.

6. Sullivan MD, Levy WC, Russo JE, Crane B, Spertus JA. Summary health status measures in advanced heart failure: relationship to clinical variables and outcome. $\mathrm{J}$ Card Fail 2007;13:560-68.

7. Heidenreich PA, Spertus JA, Jones PG, Weintraub WS, Rumsfeld JS, Rathore SS et al. Health status identifies heart failure outpatients at risk for hospitalization or death. J Am Coll Cardiol 2006;47:752-56.

8. Comin-Colet J, Garin O, Lupon J, Manito N, Crespo-Leiro MG, Gomez-Bueno M et al. Validation of the Spanish version of the Kansas city cardiomyopathy questionnaire. Rev Esp Cardiol 2011;64:51-58.

9. Comin-Colet J, Lainscak M, Dickstein K, Filippatos GS, Johnson P, Luscher TF et al. The effect of intravenous ferric carboxymaltose on health-related quality of life in patients with chronic heart failure and iron deficiency: a subanalysis of the FAIR-HF study. Eur Heart $\mathrm{J}$ 2013;34:30-38.

10. Kind P, Dolan P, Gudex C, Williams A. Variations in population health status: results from a United Kingdom national questionnaire survey. BMJ 1998;316:736-41. 
11. Anguita M, Comin-Colet J, Formiga F, Almenar M, Crespo-Leiro M, Manzano L. Tratamiento de la insuficiencia cardiaca con función sistólica deprimida: situación actual en España. Resultados del estudio VIDA-IC. Rev Esp Cardiol. 2014;67:769-70

12. Green CP, Porter CB, Bresnahan DR, Spertus JA. Development and evaluation of the Kansas City Cardiomyopathy Questionnaire: a new health status measure for heart failure. J Am Coll Cardiol 2000;35:1245-55.

13. Badia X, Roset M, Herdman M, Kind P. A comparison of United Kingdom and Spanish general population time trade-off values for EQ-5D health states. Med Decis Making 2001;21:7-16.

14. Encuesta Nacional de Salud 2001-2012 del Instituto Nacional de Estadística. Disponible en: $\underline{\text { http://www.ine.es/jaxi/menu.do?type=pcaxis } \& p a t h=/ t 15 / p 419 \& f i l e=i n e b a s e \& L=0}$

15. Mata Cases M, Roset Gamisans M, Badia Llach X, Antoñanzas Villar F, Ragel Alcazar J. Impacto de la diabetes mellitus tipo 2 en la calidad de vida de los pacientes tratados en las consultas de atención primaria en España. Atencion Primaria 2003;31:493-99.

16. Baquero M, Peset V, Burguera JA, Salazar-Cifre A, Bosca-Blasco ME, del Olmo-Rodriguez A et al. [Quality of life in Alzheimer's disease]. Rev Neurol 2009;49:337-42.

17. Miravitlles M, Soriano JB, Garcia-Rio F, Munoz L, Duran-Tauleria E, Sanchez G et al. Prevalence of COPD in Spain: impact of undiagnosed COPD on quality of life and daily life activities. Thorax 2009;64:863-68.

18. Cobo Sánchez JL, Pelayo Alonso R, Ibarguren Rodriguez E, Aja Crespo A, Saenz de Buruaga Perea A, Incera Setien ME et al. Factores sociológicos y calidad de vida relacionada con la salud en pacientes en hemodiálisis. Rev Soc Esp Enferm Nefrol 2011;14:98-104.

19. Alonso J, Ferrer M, Gandek B, Ware JE, Jr., Aaronson NK, Mosconi P et al. Health-related quality of life associated with chronic conditions in eight countries: results from the International Quality of Life Assessment (IQOLA) Project. Qual Life Res 2004;13:283-98.

20. Badia X. Estudios sobre calidad de vida de pacientes afectados por determinadas patologias. Madrid: Ministerio de Sanidad y Politica Social. 2009. Disponible en: http://www.msssi.gob.es/organizacion/sns/planCalidadSNS/docs/Estudios calidad vida pa cientes.pdf 
21. McMurray JJ, Packer M, Desai AS, Gong J, Lefkowitz M, Rizkala AR et al. Baseline characteristics and treatment of patients in Prospective comparison of ARNI with ACEI to Determine Impact on Global Mortality and morbidity in Heart Failure trial (PARADIGM-HF). Eur J Heart Fail 2014;16:817-25.

22. Ponikowski P, van Veldhuisen DJ, Comin-Colet J, Ertl G, Komajda M, Mareev $V$ et al. Beneficial effects of long-term intravenous iron therapy with ferric carboxymaltose in patients with symptomatic heart failure and iron deficiency. Eur Heart J 2014; advance access published November 20, 2014. Disponible en: http://eurheartj.oxfordjournals.org.proxy1.athensams.net/content/early/2014/11/20/eurheartj. ehu385.long.

23. Comin-Colet J, Verdu-Rotellar JM, Vela E, Cleries M, Bustins M, Mendoza L et al.Supervivencia de pacientes ambulatorios con insuficiencia cardiaca crónica del área mediterránea. Un estudio de base poblacional.Rev Esp Cardiol. 2013;66:539-44

24. Gutzwiller FS, Pfeil AM, Comin-Colet J, Ponikowski P, Filippatos G, Mori C et al. Determinants of quality of life of patients with heart failure and iron deficiency treated with ferric carboxymaltose: FAIR-HF sub-analysis. Int J Cardiol 2013;168:3878-83.

25. Enjuanes C, Klip IT, Bruguera J, Cladellas M, Ponikowski P, Banasiak W et al. Iron deficiency and health-related quality of life in chronic heart failure: results from a multicenter European study. Int J Cardiol 2014;174:268-75.

26. Comin-Colet J, Enjuanes C, Gonzalez G, Torrens A, Cladellas M, Merono O et al. Iron deficiency is a key determinant of health-related quality of life in patients with chronic heart failure regardless of anaemia status. Eur J Heart Fail 2013;15:1164-72.

27. Rodriguez-Artalejo F, Guallar-Castillon P, Pascual CR, Otero CM, Montes AO, Garcia AN et al. Health-related quality of life as a predictor of hospital readmission and death among patients with heart failure. Arch Intern Med 2005;165:1274-79.

28. Zuluaga MC, Guallar-Castillon P, Lopez-Garcia E, Banegas JR, Conde-Herrera M, OlcozChiva $\mathrm{M}$ et al. Generic and disease-specific quality of life as a predictor of long-term mortality in heart failure. Eur J Heart Fail 2010;12:1372-78.

29. Masseria C, Allin S, Sorenson C, Papanicolas I and Elias Mossialos. What are the methodological issues related to measuring healthand drawing comparisons across 
countries?. Disponible en:

http://ec.europa.eu/social/BlobServlet?docld=3951\&langld=en 
Tabla 1. Características demográficas y clínicas de todos los pacientes incluidos en el estudio y en función del nivel de calidad de vida relacionada con la salud.

\begin{tabular}{|c|c|c|c|c|}
\hline Variables & $\begin{array}{c}\text { Global } \\
(n=1.037)\end{array}$ & $\begin{array}{l}\text { Pacientes con CVRS } \\
\text { preservada }{ }^{\dagger} \\
(n=696)\end{array}$ & $\begin{array}{c}\text { Pacientes con CVRS } \\
\text { afectada } \\
(n=327)\end{array}$ & $\begin{array}{c}\text { Valor de } \\
\text { P }\end{array}$ \\
\hline Edad, años & $70,6 \pm 11,1$ & $69,2 \pm 11,2$ & $73,6 \pm 10,2$ & $<0,0001$ \\
\hline Sexo, mujeres $n(\%)$ & $309(30,1)$ & $175(25,3)$ & $129(39,9)$ & $<0,001$ \\
\hline $\mathrm{IMC} \mathrm{Kg/ \textrm {m } ^ { 2 }}$ & $27,7 \pm 3,9$ & $27,6 \pm 3,6$ & $27,9 \pm 4,5$ & 0,343 \\
\hline Tensión arterial sistólica, mmHg & $127,2 \pm 18,7$ & $127,3 \pm 17,7$ & $127 \pm 20,7$ & 0,807 \\
\hline Frecuencia Cardíaca, Ipm & $73,9 \pm 15,7$ & $73,4 \pm 15,7$ & $75,2 \pm 15,6$ & 0,09 \\
\hline Clase funcional de la NYHA I-II/III-IV, n (\%) & $550(54,9) / 452(45,1)$ & $481(71,8) / 189(28,2)$ & $59(18,5) / 260(81,5)$ & $<0,001$ \\
\hline FEVI (\%) & $33,7 \pm 6,8$ & $34,4 \pm 6,4$ & $32,2 \pm 7,5$ & $<0,0001$ \\
\hline Índice de Charlson, puntos & $4,4 \pm 2,8$ & $3,9 \pm 2,5$ & $5,2 \pm 3,1$ & $<0,0001$ \\
\hline Etiologia isquémica, $\mathrm{n}(\%)$ & $527(50,8)$ & $345(49,6)$ & $175(53,5)$ & 0,239 \\
\hline \multicolumn{5}{|l|}{ Comorbilidades } \\
\hline Hipertensión Arterial, n (\%) & $821(79,2)$ & $539(77,4)$ & $271(82,9)$ & 0,046 \\
\hline Diabetes Mellitus; n (\%) & $456(44,0)$ & $288(41,4)$ & $160(48,9)$ & 0,023 \\
\hline Insuficiencia renal significativa, n (\%) & $244(23,5)$ & $126(18,1)$ & $115(35,2)$ & $<0,001$ \\
\hline Fibrilación Auricular, n (\%) & $447(45,5)$ & $279(42,1)$ & $161(52,8)$ & 0,002 \\
\hline Anemia, n (\%) & $202(21,3)$ & $110(17,1)$ & $90(30,6)$ & $<0,001$ \\
\hline \multicolumn{5}{|l|}{ Tratamientos $\mathbf{n}(\%)$} \\
\hline IECA o ARA-II & $929(89,6)$ & $633(91,0)$ & $283(86,5)$ & 0,032 \\
\hline Betabloqueantes & $794(76,6)$ & $544(78,2)$ & $238(72,8)$ & 0,059 \\
\hline Antagonistas Aldosterona & $689(66,4)$ & $451(64,8)$ & $228(69,7)$ & 0,12 \\
\hline Ivabradina & $91(8,8)$ & $64(9,2)$ & $27(8,3)$ & 0,623 \\
\hline Digital & $225(21,7)$ & $137(19,7)$ & $85(26,0)$ & 0,022 \\
\hline Diuréticos & $925(89,2)$ & $605(86,9)$ & $306(93,6)$ & 0,001 \\
\hline Estatinas & $786(75,8)$ & $533(76,6)$ & $240(73,4)$ & 0,269 \\
\hline Antiagregantes & $622(60,0)$ & $419(60,2)$ & $193(59,0)$ & 0,72 \\
\hline Anticoagulantes & $414(39,9)$ & $253(36,4)$ & $156(47,7)$ & 0,001 \\
\hline \multicolumn{5}{|l|}{ Valores de Laboratorio } \\
\hline Hemoglobina, g/dL & $12,9 \pm 1,7$ & $13 \pm 1,6$ & $12,5 \pm 1,7$ & $<0,0001$ \\
\hline TFGe-ml/min $/ 1,73 \mathrm{~m}^{2}$ & $61,2 \pm 27,6$ & $64,9 \pm 27,7$ & $53,7 \pm 26,2$ & $<0,0001$ \\
\hline
\end{tabular}


Aclaramiento de creatinina $<60, \mathrm{n}(\%)$

NT-proBNP, pg/mL

BNP, $(\mathrm{pg} / \mathrm{mL})$

$1854,1 \pm 1829,8$
$515 \pm 1871,8$

$138(36,4)$

$1560,2 \pm 1361,6$

$117(63,2)$

$2491,6 \pm 2489,2$

$341 \pm 280,1$

0,001

$616,2 \pm 2342,8$

50 puntos. 
Tabla 2. Distribución de las puntuaciones sumarias, de las dimensiones y de los diversos dominios de los cuestionarios de calidad de vida específico para IC (KCCQ) y genérico usados en toda la población estudiada y en función del nivel de calidad de vida relacionada con la salud.

\begin{tabular}{|c|c|c|c|c|}
\hline KCCQ, subdominios & $\begin{array}{c}\text { Global } \\
(n=1.037)\end{array}$ & $\begin{array}{c}\text { Pacientes con CVRS } \\
\text { preservada }{ }^{\dagger} \\
(n=696) \\
\end{array}$ & $\begin{array}{c}\text { Pacientes con CVRS } \\
\text { afectada } \\
(n=327) \\
\end{array}$ & $\begin{array}{c}\text { Valor de } \\
\text { P }\end{array}$ \\
\hline Limitación Física (media $\pm D E$ ) & $61,1 \pm 28,1$ & $75,7 \pm 18,2$ & $29,9 \pm 18,1$ & $<0,0001$ \\
\hline Estabilidad de los Síntomas & $59,5 \pm 23,2$ & $63 \pm 21,2$ & $51,9 \pm 25,4$ & $<0,0001$ \\
\hline Frecuencia de los Síntomas & $66,3 \pm 26,1$ & $79,9 \pm 15,3$ & $37,1 \pm 19,2$ & $<0,0001$ \\
\hline Carga de los Síntomas & $67,1 \pm 26,1$ & $80,7 \pm 16$ & $37,5 \pm 17,6$ & $<0,0001$ \\
\hline Autoeficacia & $69,1 \pm 22,5$ & $72,9 \pm 20,2$ & $60,6 \pm 24,6$ & $<0,0001$ \\
\hline Calidad de Vida & $54,4 \pm 24,1$ & $66,6 \pm 16,6$ & $28,1 \pm 14,4$ & $<0,0001$ \\
\hline Limitación Social & $61,6 \pm 29,4$ & $77,7 \pm 17,9$ & $27,3 \pm 17$ & $<0,0001$ \\
\hline \multicolumn{5}{|l|}{$K C C Q$, medidas sumarias } \\
\hline Puntuación Sumaria Global & $60,9 \pm 24,5$ & $75,1 \pm 13,5$ & $30,6 \pm 12,3$ & $<0,0001$ \\
\hline Puntuación Sumaria Clínica & $63,9 \pm 25,2$ & $78 \pm 14,4$ & $33,6 \pm 14,4$ & $<0,0001$ \\
\hline Puntuación Sumaria de Síntomas & $66,7 \pm 25,4$ & $80,3 \pm 15$ & $37,3 \pm 17$ & $<0,0001$ \\
\hline \multicolumn{5}{|c|}{ EQ-5D, \% pacientes reportando problemas } \\
\hline Movilidad, n (\%) & $586(58,1)$ & $273(40,7)$ & $304(93,5)$ & $<0,001$ \\
\hline Cuidado Personal, n (\%) & $382(38,0)$ & $132(19,7)$ & $246(76,4)$ & $<0,001$ \\
\hline Actividades Cotidianas, n (\%) & $619(61,4)$ & $307(45,8)$ & $305(93,8)$ & $<0,001$ \\
\hline Dolor/ Malestar, n (\%) & $510(50,6)$ & $256(38,1)$ & $248(76,8)$ & $<0,001$ \\
\hline Ansiedad/Depresión, n (\%) & $493(48,9)$ & $237(35,3)$ & $249(76,9)$ & $<0,001$ \\
\hline \multicolumn{5}{|l|}{ EQ-5D, medidas sumarias } \\
\hline Índice EQ-5D global (media $\pm D E$ ) & $0,6 \pm 0,3$ & $0,8 \pm 0,2$ & $0,4 \pm 0,2$ & $<0,0001$ \\
\hline 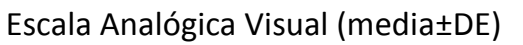 & $60,8 \pm 20$ & $68,7 \pm 15,8$ & $43,5 \pm 16,8$ & $<0,0001$ \\
\hline
\end{tabular}

tCVRS: Calidad de Vida Relacionada con la Salud. Se definió CVRS preservada cuando la puntuación sumaria global del KCCQ fue $\geq 50$ puntos. 
Tabla 3. Matriz de correlaciones (valores de R e intervalos de confianza) entre los diferentes ítems, dimensiones, dominios y puntuaciones sumarias de los cuestionarios para evaluar la CVRS usados en el presente estudio. * La correlación tiene un $\mathrm{p}$-valor=0,004; en el resto de coeficientes de correlación presentados, p<0,0001

\begin{tabular}{|c|c|c|c|c|c|c|c|c|c|c|c|c|}
\hline & [1] & [2] & [3] & [4] & [5] & [6] & [7] & [8] & [9] & [10] & [11] & [12] \\
\hline Puntación EQ-5D [1] & $\begin{array}{c}1,000 \\
-\end{array}$ & & & & & & & & & & & \\
\hline Escala visual analógica [2] & $\begin{array}{c}0,682 \\
(0,647-0,714)\end{array}$ & $\begin{array}{c}1,000 \\
-\end{array}$ & & & & & & & & & & \\
\hline Limitación física [3] & $\begin{array}{c}0,785 \\
(0,759-0,807)\end{array}$ & $\begin{array}{c}0,634 \\
(0,596-0,669)\end{array}$ & $\begin{array}{c}1,000 \\
-\end{array}$ & & & & & & & & & \\
\hline Síntomas de estabilidad [4] & $\begin{array}{c}0,207 \\
(0,147-0,266)\end{array}$ & $\begin{array}{c}0,229 \\
(0,17-0,286)\end{array}$ & $\begin{array}{c}0,176 \\
(0,116-0,235)\end{array}$ & $\begin{array}{c}1,000 \\
-\end{array}$ & & & & & & & & \\
\hline Frecuencia de los síntomas [5] & $\begin{array}{c}0,717 \\
(0,685-0,745)\end{array}$ & $\begin{array}{c}0,601 \\
(0,56-0,639)\end{array}$ & $\begin{array}{c}0,756 \\
(0,729-0,781)\end{array}$ & $\begin{array}{c}0,203 \\
(0,144-0,261)\end{array}$ & $\begin{array}{c}1,000 \\
-\end{array}$ & & & & & & & \\
\hline Carga de síntomas [6] & $\begin{array}{c}0,721 \\
(0,689-0,749) \\
\end{array}$ & $\begin{array}{c}0,633 \\
(0,595-0,669) \\
\end{array}$ & $\begin{array}{c}0,756 \\
(0,729-0,781) \\
\end{array}$ & $\begin{array}{c}0,240 \\
(0,182-0,297) \\
\end{array}$ & $\begin{array}{c}0,903 \\
(0,891-0,914) \\
\end{array}$ & $\begin{array}{c}1,000 \\
- \\
\end{array}$ & & & & & & \\
\hline $\begin{array}{l}\text { Puntuación total de síntomas } \\
\text { (TSS) [7] }\end{array}$ & $\begin{array}{c}0,736 \\
(0,706-0,763)\end{array}$ & $\begin{array}{c}0,633 \\
(0,594-0,668)\end{array}$ & $\begin{array}{c}0,775 \\
(0,749-0,798)\end{array}$ & $\begin{array}{c}0,227 \\
(0,168-0,284)\end{array}$ & $\begin{array}{c}0,976 \\
(0,972-0,978)\end{array}$ & $\begin{array}{c}0,976 \\
(0,972-0,978)\end{array}$ & $\begin{array}{c}1,000 \\
-\end{array}$ & & & & & \\
\hline Autoeficacia [8] & $\begin{array}{c}0,349 \\
(0,293-0,402)\end{array}$ & $\begin{array}{c}0,301 \\
(0,244-0,356)\end{array}$ & $\begin{array}{c}0,322 \\
(0,266-0,375)\end{array}$ & $\begin{array}{c}0,0909 * \\
(0,03-0,151)\end{array}$ & $\begin{array}{c}0,340 \\
(0,284-0,392)\end{array}$ & $\begin{array}{c}0,345 \\
(0,29-0,398)\end{array}$ & $\begin{array}{c}0,351 \\
(0,296-0,403)\end{array}$ & $\begin{array}{c}1,000 \\
-\end{array}$ & & & & \\
\hline Calidad de vida [9] & $\begin{array}{c}0,701 \\
(0,668-0,731) \\
\end{array}$ & $\begin{array}{c}0,629 \\
(0,591-0,665) \\
\end{array}$ & $\begin{array}{c}0,695 \\
(0,662-0,726) \\
\end{array}$ & $\begin{array}{c}0,210 \\
(0,151-0,268) \\
\end{array}$ & $\begin{array}{c}0,779 \\
(0,754-0,802) \\
\end{array}$ & $\begin{array}{c}0,788 \\
(0,763-0,81) \\
\end{array}$ & $\begin{array}{c}0,802 \\
(0,78-0,823) \\
\end{array}$ & $\begin{array}{c}0,318 \\
(0,262-0,372) \\
\end{array}$ & $\begin{array}{c}1,000 \\
- \\
\end{array}$ & & & \\
\hline Limitaciones sociales [10] & $\begin{array}{c}0,751 \\
(0,723-0,777)\end{array}$ & $\begin{array}{c}0,645 \\
(0,607-0,679)\end{array}$ & $\begin{array}{c}0,822 \\
(0,801-0,841)\end{array}$ & $\begin{array}{c}0,208 \\
(0,149-0,266)\end{array}$ & $\begin{array}{c}0,790 \\
(0,766-0,812)\end{array}$ & $\begin{array}{c}0,795 \\
(0,772-0,817)\end{array}$ & $\begin{array}{c}0,812 \\
(0,79-0,832)\end{array}$ & $\begin{array}{c}0,300 \\
(0,243-0,354)\end{array}$ & $\begin{array}{c}0,815 \\
(0,793-0,834)\end{array}$ & $\begin{array}{c}1,000 \\
-\end{array}$ & & \\
\hline $\begin{array}{l}\text { Medida resumen total (OSS) } \\
\text { [11] }\end{array}$ & $\begin{array}{c}0,815 \\
(0,792-0,834)\end{array}$ & $\begin{array}{c}0,698 \\
(0,665-0,729)\end{array}$ & $\begin{array}{c}0,905 \\
(0,893-0,916)\end{array}$ & $\begin{array}{c}0,224 \\
(0,165-0,281)\end{array}$ & $\begin{array}{c}0,897 \\
(0,884-0,908) \\
\end{array}$ & $\begin{array}{c}0,903 \\
(0,891-0,914)\end{array}$ & $\begin{array}{c}0,921 \\
(0,912-0,93)\end{array}$ & $\begin{array}{c}0,352 \\
(0,297-0,404) \\
\end{array}$ & $\begin{array}{c}0,898 \\
(0,885-0,909) \\
\end{array}$ & $\begin{array}{c}0,944 \\
(0,937-0,951) \\
\end{array}$ & $\begin{array}{c}1,000 \\
-\end{array}$ & \\
\hline $\begin{array}{l}\text { Medida resumen clínica (CSS) } \\
\text { [12] }\end{array}$ & 0,807 & 0,672 & 0,948 & 0,211 & 0,913 & 0,913 & 0,936 & 0,358 & 0,792 & 0,866 & 0,968 & 1,000 \\
\hline
\end{tabular}


Tabla 4. Modelos univariantes y multivariados de regresión lineal para la evaluación de los factores demográficos y clínicos asociados a la CVRS medida mediante la puntuación sumaria del Kansas City Cardiomyopathy Questionnaire (KCCQ OSS), índice global del EuroQoL de 5 dimensiones (EQ-5D index) y la Escala Analógica Visual del EQ-5D (EAV).

\begin{tabular}{|c|c|c|c|c|c|c|c|c|c|c|c|c|c|c|c|}
\hline \multirow{3}{*}{$\begin{array}{l}\text { Modelos } \\
\text { Médidas de CVRS } \\
\text { Determinantes }\end{array}$} & \multicolumn{9}{|c|}{ Univariantes } & \multicolumn{6}{|c|}{ Multivariantes (pasos atrás) } \\
\hline & \multicolumn{3}{|c|}{ KCCQ OSS } & \multicolumn{3}{|c|}{ EQ-5D index } & \multicolumn{3}{|c|}{ EAV } & \multicolumn{2}{|c|}{ KCCQ OSS } & \multicolumn{2}{|c|}{ EQ-5D index } & \multicolumn{2}{|c|}{ EAV } \\
\hline & $c \beta E^{*}$ & $R^{2}$ & Valor $p$ & $c \beta E^{*}$ & $\mathbf{R}^{2}$ & Valor $p$ & $c \beta E^{*}$ & $\mathbf{R}^{2}$ & Valor $p$ & $c \beta E^{*}$ & Valor $p$ & $c \beta E^{*}$ & Valor $\mathbf{p}$ & $c \beta E^{*}$ & Valor $p$ \\
\hline Edad, 1 año & $-0,275$ & 0,076 & $<0,001$ & $-0,287$ & 0,082 & $<0,001$ & $-0,223$ & 0,050 & $<0,001$ & $-0,230$ & 0,030 & $-0,004$ & 0,002 & $-0,178$ & 0,072 \\
\hline Sexo, hombres/mujeres & $-0,157$ & 0,025 & $<0,001$ & $-0,169$ & 0,029 & $<0,001$ & $-0,108$ & 0,012 & 0,001 & $-10,258$ & $<0,001$ & $-0,105$ & $<0,001$ & $-3,683$ & 0,095 \\
\hline IMC, 1 Kg/m2 & $-0,056$ & 0,003 & 0,077 & $-0,082$ & 0,007 & 0,010 & $-0,016$ & 0,000 & 0,610 & & & & & & \\
\hline TA Sistólica, 1 mmHg & $-0,010$ & 0,000 & 0,743 & 0,001 & 0,000 & 0,965 & 0,041 & 0,002 & 0,194 & & & & & & \\
\hline Frecuencia Cardíaca, 1 Ipm & $-0,103$ & 0,011 & 0,001 & $-0,093$ & 0,009 & 0,003 & $-0,059$ & 0,004 & 0,059 & & & & & & \\
\hline Clase funcional NYHA, I-II/III-IV & $-0,562$ & 0,316 & $<0,001$ & $-0,465$ & 0,216 & $<0,001$ & $-0,453$ & 0,206 & $<0,001$ & $-20,373$ & $<0,001$ & $-0,180$ & $<0,001$ & $-12,586$ & $<0,001$ \\
\hline FEVI, 1 punto $\%$ & 0,156 & 0,024 & $<0,001$ & 0,129 & 0,017 & $<0,001$ & 0,165 & 0,027 & $<0,001$ & 0,254 & 0,135 & & & 0,263 & 0,086 \\
\hline Índice de Charlson, 1 punto & $-0,285$ & 0,081 & $<0,001$ & $-0,318$ & 0,101 & $<0,001$ & $-0,240$ & 0,058 & $<0,001$ & $-1,258$ & 0,005 & $-0,008$ & 0,136 & $-1,029$ & 0,009 \\
\hline Etiología isquémica, no/si & $-0,068$ & 0,005 & 0,030 & $-0,086$ & 0,007 & 0,006 & $-0,050$ & 0,003 & 0,114 & & & $-0,053$ & 0,055 & & \\
\hline TFGe, $1 \mathrm{ml} / \mathrm{min} / 1.73 \mathrm{~m} 2$ & 0,193 & 0,037 & $<0,001$ & 0,187 & 0,035 & $<0,001$ & 0,173 & 0,030 & $<0,001$ & & & & & & \\
\hline Hipertensión arterial, no/si & $-0,097$ & 0,010 & 0,002 & $-0,126$ & 0,016 & $<0,001$ & $-0,040$ & 0,002 & 0,202 & & & $-0,060$ & 0,085 & & \\
\hline Fibrilación Auricular, no/si & $-0,152$ & 0,023 & $<0,001$ & $-0,172$ & 0,030 & $<0,001$ & $-0,145$ & 0,021 & $<0,001$ & & & & & & \\
\hline $\mathrm{DM}, \mathrm{no} / \mathrm{si}$ & $-0,149$ & 0,022 & $<0,001$ & $-0,164$ & 0,027 & $<0,001$ & $-0,106$ & 0,011 & 0,001 & & & $-0,041$ & 0,140 & & \\
\hline Hemoglobina, $1 \mathrm{~g} / \mathrm{dL}$ & 0,227 & 0,051 & $<0,001$ & 0,245 & 0,060 & $<0,001$ & 0,214 & 0,046 & $<0,001$ & & & & & 1,023 & 0,087 \\
\hline Tratamiento óptimo, no/si & 0,028 & 0,001 & 0,366 & 0,042 & 0,002 & 0,188 & 0,038 & 0,001 & 0,230 & & & & & & \\
\hline Servicio de inclusión, CAR/MI & $-0,186$ & 0,035 & $<0,001$ & $-0,197$ & 0,039 & $<0,001$ & $-0,185$ & 0,034 & $<0,001$ & $-4,595$ & 0,049 & $-0,035$ & 0,185 & $-4,761$ & 0,022 \\
\hline Ingreso reciente, si/no & 0,259 & 0,067 & $<0,001$ & 0,201 & 0,041 & $<0,001$ & 0,195 & 0,038 & $<0,001$ & 6,286 & 0,006 & 0,046 & 0,075 & & \\
\hline Tiempo desde diagnóstico $<1$ año no/si & $-0,070$ & 0,005 & 0,034 & $-0,067$ & 0,004 & 0,046 & $-0,072$ & 0,005 & 0,029 & & & & & & \\
\hline
\end{tabular}


${ }^{*} \mathrm{c} \beta \mathrm{E}$ : Coeficiente $\beta$ Estandarizado. En las variables dicotómicas, la primera es la categoría de referencia.

Abreviaturas: IMC (índice de masa corporal), TA (tensíon arterial), NYHA (New York Heart Association), FEVI (fracción de eyección del ventrículo izquierdo, TGFe (tasa de filtrado glomerular estimada), DM (diabetes mellitus) CAR/MI (Cardiología/Medicina Interna) 
Tabla 5. Análisis no ajustado de las puntuaciones medias del sumario global del KCCQ, la Escala Analógica Visual y del índice del EQ-5D en los subgrupos de pacientes que mostraron asociación independiente con la puntuación sumaria del KCCQ en el análisis de regresión lineal multivariado. Se representa la media \pm desviación estándar en función del sexo (hombres vs. mujeres), clase funcional de la NYHA (I-II vs. III-IV), edad ( $<75$ años vs. $\geq 75$ años), índice de comorbilidad de Charlson ( $\leq 4$ [mediana] vs. >4), tiempo desde el último ingreso (<30 días vs. $\geq 30$ días) y servicio clínico responsable (Cardiología vs. Medicina Interna). *Valor de $\mathbf{P}<0,05$.

\begin{tabular}{|c|c|c|c|c|c|c|c|c|c|c|c|c|}
\hline & \multicolumn{2}{|c|}{ Sexo } & \multicolumn{2}{|c|}{ NYHA } & \multicolumn{2}{|c|}{ Edad (años) } & \multicolumn{2}{|c|}{$\begin{array}{l}\text { Comorbilidad } \\
\text { Índice Charlson }\end{array}$} & \multicolumn{2}{|c|}{ Servicio } & \multicolumn{2}{|c|}{ Hospitalización Reciente } \\
\hline & $\begin{array}{l}\text { Hombres } \\
(n=719)\end{array}$ & $\begin{array}{c}\text { Mujeres } \\
(n=309)\end{array}$ & $\begin{array}{c}I-I I \\
(n=550)\end{array}$ & $\begin{array}{c}\text { III-IV } \\
(n=452)\end{array}$ & $\begin{array}{c}<75 \\
(n=601)\end{array}$ & $\begin{array}{c}\geq 75 \\
(n=418)\end{array}$ & $\begin{array}{c}\leq 4 \\
(n=324)\end{array}$ & $\begin{array}{c}>4 \\
(n=207)\end{array}$ & $\begin{array}{c}\text { Cardiología } \\
\text { (n=638) }\end{array}$ & $\begin{array}{l}\text { Medicina } \\
\text { Interna } \\
(n=367)\end{array}$ & $\begin{array}{c}\text { No } \\
(n=647)\end{array}$ & $\begin{array}{c}S i \\
(n=386)\end{array}$ \\
\hline $\begin{array}{l}\text { KCCQ OSS } \\
\text { (media, desviación típica) }\end{array}$ & $\begin{array}{c}63,4 \\
(24,0)\end{array}$ & $\begin{array}{l}55,0 \\
(24,6)\end{array}$ & $\begin{array}{c}73,4 \\
(18,8)\end{array}$ & $\begin{array}{c}45,6 \\
(22,0)\end{array}$ & $\begin{array}{c}64,8 \\
(24,2)\end{array}$ & $\begin{array}{c}55,2 \\
(23,9)\end{array}$ & $\begin{array}{c}62,5 \\
(23,8)\end{array}$ & $\begin{array}{c}54,5 \\
(23,9)\end{array}$ & $\begin{array}{c}66,1 \\
(22,2)\end{array}$ & $\begin{array}{l}56,6 \\
(23,9)\end{array}$ & $\begin{array}{c}65,3 \\
(23,6)\end{array}$ & $\begin{array}{l}53,7 \\
(24,5)\end{array}$ \\
\hline $\begin{array}{l}\text { Escala visual analógica } \\
\text { (media, desviación típica) }\end{array}$ & $\begin{array}{c}62,2 \\
(19,4)\end{array}$ & $\begin{array}{c}57,5 \\
(20,8)\end{array}$ & $\begin{array}{c}68,9 \\
(16,6)\end{array}$ & $\begin{array}{c}50,7 \\
(19,1)\end{array}$ & $\begin{array}{c}63,5 \\
(19,7)\end{array}$ & $\begin{array}{c}56,9 \\
(19,9)\end{array}$ & $\begin{array}{c}61,6 \\
(20,3)\end{array}$ & $\begin{array}{c}56,5 \\
(20,1)\end{array}$ & $\begin{array}{c}63,7 \\
(18,6)\end{array}$ & $\begin{array}{c}56,0 \\
(23,9)\end{array}$ & $\begin{array}{c}63,1 \\
(19,9)\end{array}$ & $\begin{array}{c}57,1 \\
(19,7)\end{array}$ \\
\hline $\begin{array}{l}\text { Índice EQ-5D } \\
\text { (media, desviación típica) }\end{array}$ & $\begin{array}{c}0,7 \\
(0,3)\end{array}$ & $\begin{array}{c}0,6 \\
(0,3)\end{array}$ & $\begin{array}{c}0,8 \\
(0,2)\end{array}$ & $\begin{array}{c}0,5 \\
(0,2)\end{array}$ & $\begin{array}{c}0,7 \\
(0,3)\end{array}$ & $\begin{array}{c}0,6 \\
(0,2)\end{array}$ & $\begin{array}{c}0,7 \\
(0,3)\end{array}$ & $\begin{array}{c}0,6 \\
(0,3)\end{array}$ & $\begin{array}{l}0,7 \\
(0,2)\end{array}$ & $\begin{array}{c}0,6 \\
(0,3)\end{array}$ & $\begin{array}{l}0,7 \\
(0,2)\end{array}$ & $\begin{array}{c}0,6 \\
(0,3)\end{array}$ \\
\hline
\end{tabular}

Todos los valores de $\mathrm{p}$ para las diferencias entre grupos fueron $<0,05$ 


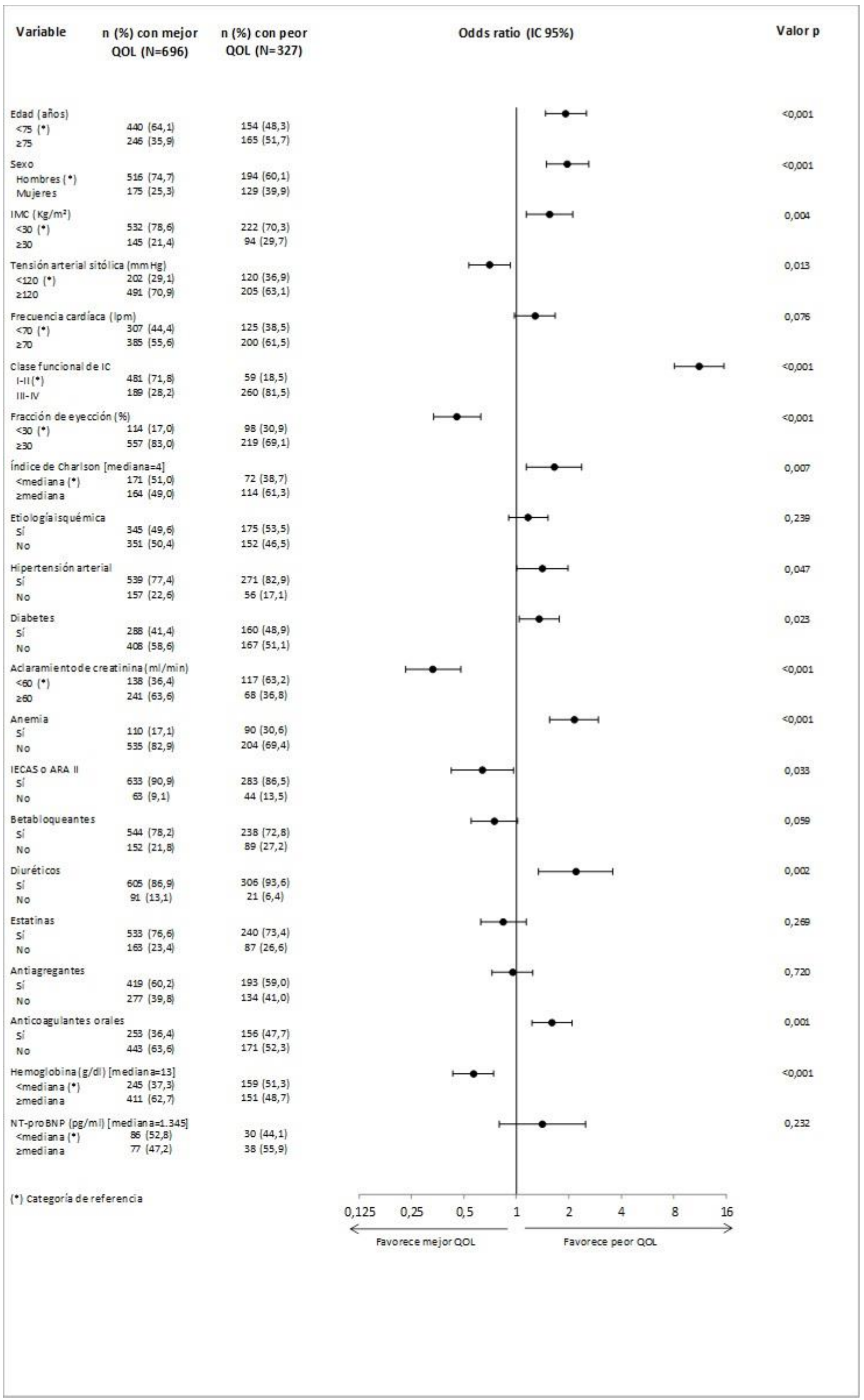




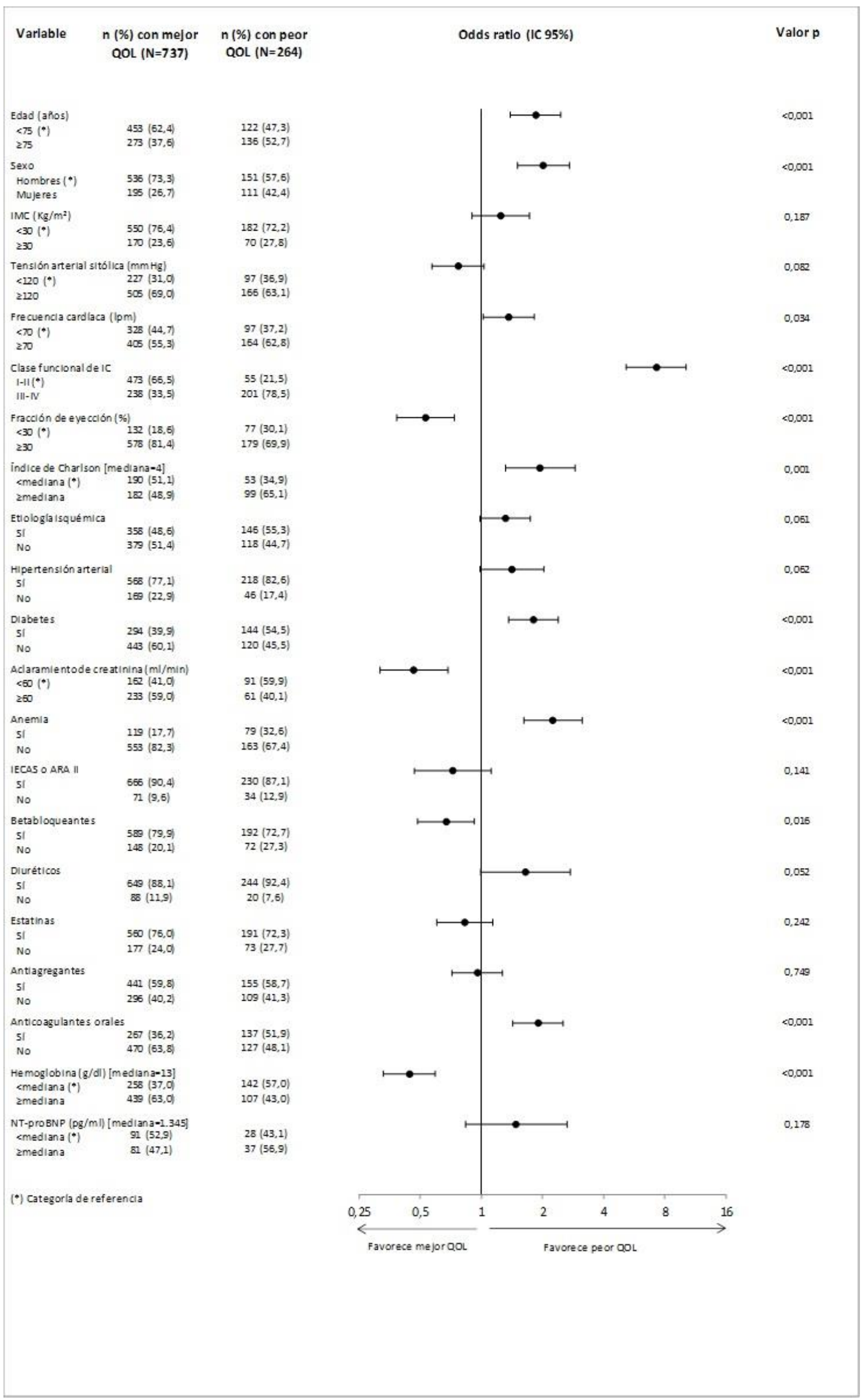




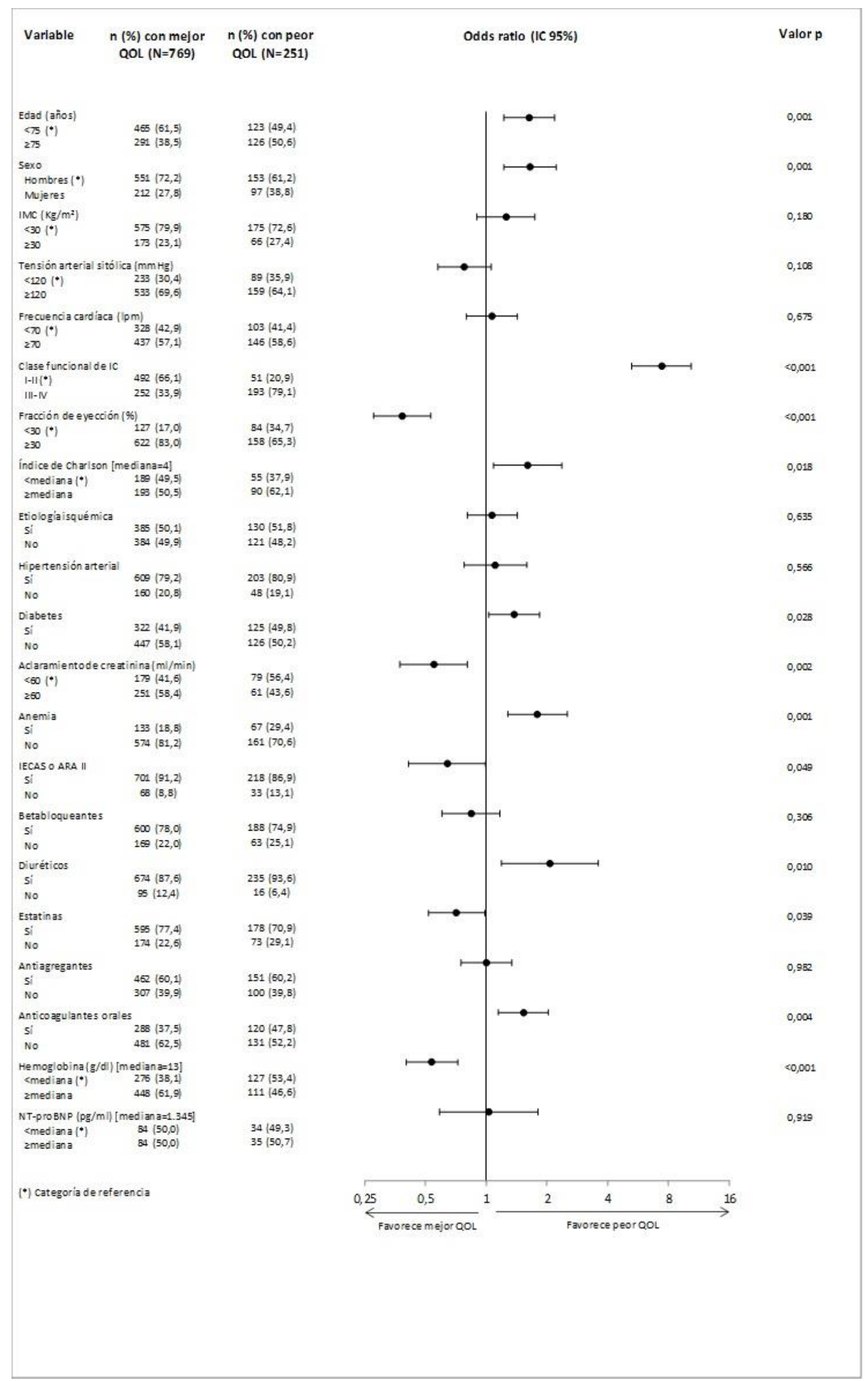

